\title{
Careful consideration should be paid in the new imaging modality evaluation
}

\author{
Tetsuro Sekine ${ }^{1}$, Masatoki Nakaza ${ }^{2}$, Shinichiro Kumita ${ }^{2}$ \\ ${ }^{1}$ Department of Radiology, Nippon Medical School Musashi-Kosugi Hospital, Kanagawa, Japan; ${ }^{2}$ Department of Radiology, Nippon Medical School, \\ Tokyo, Japan \\ Correspondence to: Tetsuro Sekine, MD, PhD. Department of Radiology, Nippon Medical School Musashi-Kosugi Hospital, 1-396 Kosugi-machi, \\ Nakahara-ku, Kawasaki-shi, Kanagawa 211-8533, Japan. Email: tetsuro.sekine@gmail.com. \\ Comment on: Umehara T, Takumi K, Ueda K, et al. Four-dimensional flow magnetic resonance imaging study to explain high prevalence of \\ pulmonary vein stump thrombus after left upper lobectomy. J Thorac Dis 2020;12:5542-51.
}

Submitted Nov 04, 2020. Accepted for publication Dec 04, 2020.

doi: $10.21037 /$ jtd-20-3229

View this article at: http://dx.doi.org/10.21037/jtd-20-3229

We have read the interesting report by Umehara et al., in which they sought to clarify the specific blood flow pattern after left upper lobectomy (LUL) (1). LUL is an independent risk factor for thrombosis after lobectomy. In these patients, four-dimensional flow magnetic resonance imaging (4D flow MRI) was expected to illuminate the flow dynamics that cause thrombosis formation. However, we found insufficient or incorrect points in each section of the manuscript, which included the scan scheme, postprocessing evaluation, definition of altered flow in the left atrium (LA), and the list of referenced papers. Particularly, the definition of the blood flow pattern and turbulent blood flow pattern that prevented/promoted thrombosis formation, the central concept of this paper, could not be considered acceptable based on the vast body of evidence on LA evaluation using 4D flow MRI. We believe that this paper cannot be used as a reference for further analysis in this field for the reasons that follow. When applying a new imaging modality, we should carefully compare previous results to those currently obtained.

First, the heart phase and temporal resolution were too small [13] and too low, respectively. The consensus statement on 4D flow MRI recommended that the temporal resolution should be $<40 \mathrm{msec}$ (2). In this paper, the temporal resolution $(62.4 \mathrm{msec})$ was too low to evaluate the LA flow pattern. With these parameters, the standard deviation of the velocity is likely to be highly underestimated. Regarding this point, we noticed a strange presentation within the paper. We agree that the heart phase can change depending on the individual patients' heart rate; however, on figure 2 in the author's paper, the orange colored patient seemed to have a heart phase of eight in the LUL presentation, while, in other pulmonary veins (PVs), it was 10.

Second, the velocity measured in the region of interest (ROI) was vulnerable to even subtle differences in the position setting. We experimentally set four ROIs in the left superior PV (LSPV) after LUL and measured the velocity (Figure 1A,B). The velocity curves and standard deviations differed greatly among the ROIs (standard deviations of the velocity were 0.42 and $1.11 \mathrm{~cm} / \mathrm{sec}$ in the light blue and light green ROIs, respectively; Figure 1C). ROI measurement can be used for laminar flow such as that in normal PVs. However, with turbulent flow, ROI measurement cannot accurately capture the blood flow velocity (3). To mitigate these errors, volume of interest measurement is recommended (4-7).

Third, the authors defined the thrombus-promoting/preventing flow without any prior evidence. In general, the "vortex" in the LA is thought to prevent thrombosis, though this concept is still under discussion $(4,8)$. Why were these definitions not included in the paper? As this was the central concept of the study, clarification was required.

Fourth, a higher standard deviation does not mean turbulence. When we set the ROI at the center of the LA (purple ROI, Figure 1A,B), the standard deviation was much higher than that of the PVs $(5.23 \mathrm{~cm} / \mathrm{sec}$, Figure 1D). When the average velocity increased, generally, so did the 

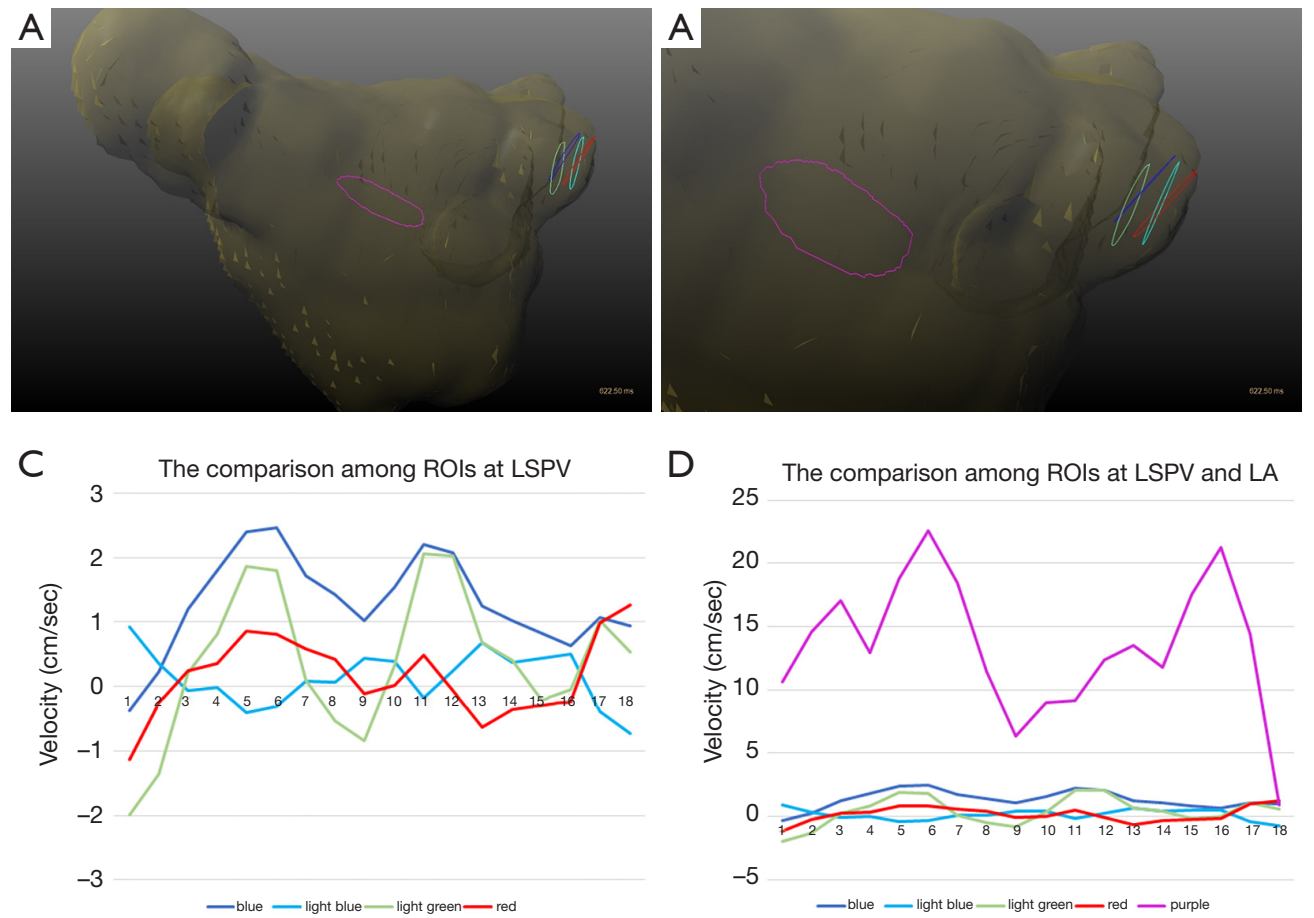

Figure 1 The representative ROIs in the LSPV (blue, light blue, green, and red) and the center of the LA [purple; (A) whole view of the LA; and (B) enlarged view of the LSPV] in the patients after LUL. (C,D) The flow curve during one R-R interval where the line color corresponds to that of the respective ROI. ROI, region of interest; LSPV, left superior pulmonary vein; LA, left atrium; LUL, left upper lobectomy.

standard deviation. The correlation between pulmonary venous stump length and the standard deviation of the velocity simply means that the average velocity increases proportionally to the distance from the wall. In almost all papers on 4D flow MRI that evaluated LA thrombus formation, decreased velocity and stasis were used as altered flow markers (4-7). This paper stated the opposite results: higher standard deviations were derived from higher velocities. We suggest that the authors clarify the rationale for this evaluation.

Fifth, the authors did not cite any major papers that evaluated LA flow using 4D flow MRI (4-7). Thus, it is apparently inadequate as a scientific paper. We agree that previous reports focused on another entity, atrial fibrillation. However, the pathophysiology of thrombosis is likely to be similar.

Finally, this is not "the first report to evaluate the dynamic blood movement to clarify the risk of PV stump thrombus by 4D-flow MRI". A case report that focused on this phenomenon was published on February 13, 2020, 2 months before this paper was submitted (April 07,
2020) (9). This description is scientifically incorrect. The authors should rectify this statement and reference this case report.

Aside from the issues with the main contents of this paper, we recommend the use of the term "4D Flow" or "4D flow" not "4D-Flow" because a hyphen may have negative effects in the keyword-search algorithm.

\section{Acknowledgments}

Funding: None.

\section{Footnote}

Provenance and Peer Review: This article was a free submission to the journal. The article did not undergo external peer review.

Conflicts of Interest: All authors have completed the ICMJE uniform disclosure form (available at http://dx.doi. org/10.21037/jtd-20-3229). The authors have no conflicts 
of interest to declare.

Ethical Statement: The authors are accountable for all aspects of the work in ensuring that questions related to the accuracy or integrity of any part of the work are appropriately investigated and resolved.

Open Access Statement: This is an Open Access article distributed in accordance with the Creative Commons Attribution-NonCommercial-NoDerivs 4.0 International License (CC BY-NC-ND 4.0), which permits the noncommercial replication and distribution of the article with the strict proviso that no changes or edits are made and the original work is properly cited (including links to both the formal publication through the relevant DOI and the license). See: https://creativecommons.org/licenses/by-nc-nd/4.0/.

\section{References}

1. Umehara T, Takumi K, Ueda K, et al. Four-dimensional flow magnetic resonance imaging study to explain high prevalence of pulmonary vein stump thrombus after left upper lobectomy. J Thorac Dis 2020;12:5542-51.

2. Dyverfeldt P, Bissell M, Barker AJ, et al. 4D flow cardiovascular magnetic resonance consensus statement. J Cardiovasc Magn Reson 2015;17:72.

3. Sugiyama M, Takehara Y, Kawate $M$, et al. Optimal plane selection for measuring post-prandial blood flow increase

Cite this article as: Sekine T, Nakaza M, Kumita S. Careful consideration should be paid in the new imaging modality evaluation. J Thorac Dis 2021;13(1):422-424. doi: 10.21037/jtd20-3229 within the superior mesenteric artery: analysis using 4D flow and computational fluid dynamics. Magn Reson Med Sci 2020;19:366-74.

4. Garcia J, Sheitt H, Bristow MS, et al. Left atrial vortex size and velocity distributions by 4D flow MRI in patients with paroxysmal atrial fibrillation: Associations with age and CHA2 DS2 -VASc risk score. J Magn Reson Imaging 2020;51:871-84.

5. Lee DC, Markl M, Ng J, et al. Three-dimensional left atrial blood flow characteristics in patients with atrial fibrillation assessed by 4D flow CMR. Eur Heart J Cardiovasc Imaging 2016;17:1259-68.

6. Markl M, Lee DC, Ng J, et al. Left atrial 4-dimensional flow magnetic resonance imaging: stasis and velocity mapping in patients with atrial fibrillation. Invest Radiol 2016;51:147-54.

7. Markl M, Lee DC, Furiasse N, et al. Left atrial and left atrial appendage 4D blood flow dynamics in atrial fibrillation. Circ Cardiovasc Imaging 2016;9:e004984.

8. Kilner PJ, Yang GZ, Wilkes AJ, et al. Asymmetric redirection of flow through the heart. Nature 2000;404:759-61.

9. Matsumoto M, Takegahara K, Inoue T, et al. 4D flow MR imaging reveals a decrease of left atrial blood flow in a patient with cardioembolic cerebral infarction after pulmonary left upper lobectomy. Magn Reson Med Sci 2020;19:290-3. 